\title{
EL ANÁLISIS DE ACTIVIDADES COMO MÉTODO DE INVESTIGACIÓN
}

THE ANALYSIS OF ACTIVITIES AS RESEARCH METHOD

Miguel Alejandro Bustamante Ubilla *1.

1 Facultad de Economía y Negocios, Universidad de Talca, Campus Lircay, Avenida Lircay S/N, Talca - Chile. E-mail: mabu@utalca.cl.

RESUMEN

En este trabajo se analiza la relación que, en términos metodológicos, existe entre el análisis de actividades y el desarrollo de nuevas prácticas de investigación. Los conceptos utilizados permiten fundamentar algunos cambios epistemológicos que conlleva la innovación en procesos de investigación que posean características complejas y se analizan algunas consideraciones éticas y algunos aspectos metodológicos que conlleva este tipo de investigación para la movilización de los recursos personales. Se trata el tema desde la definición de un problema de investigación y la influencia del método en la definición de las hipótesis y los objetivos de investigación. Se describen las fases de investigación que derivan de esta nueva metodología, el impacto que tiene esta propuesta sobre las técnicas de recogida de datos así como la incidencia sobre el análisis y evaluación de resultados. Termina con la descripción de resultados esperados en este tipo de investigaciones, reflexiones acerca de la aplicabilidad en el contexto de la formación por competencias así como la protección de los resultados del análisis de actividades.

Palabras clave: Análisis de actividades, actividades de investigación, aprender a aprender, competencias en investigación.

\section{ABSTRACT}

This paper analyzes the relationship in terms of methodology, between analysis activities and development of new research practices. The concepts used allow epistemological base changes involved in innovation in research processes that have complex features and analyzes some ethical and methodological aspects involved in this type of research for the mobilization of personal resources. This is the theme from the definition of a research problem and the influence of the method in the definition of hypotheses and research objectives. It describes the stages of research stemming from this new methodology, the impact of this proposal on data collection techniques and the impact on analysis and evaluation of results. It ends with the description of expected results in this type of research, reflections on the applicability in the context of skills training and the protection of the results of activity analysis.

Keywords: Analysis of activities, investigation activities, to learn how to learn, competitions in investigation. 


\section{INTRODUCCIÓN}

En la actualidad parece evidente que la re-

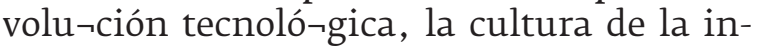
formación y la comunica-ción so-cial, además de la interconectividad de las personas hacen de la sociedad una realidad compleja. Por estas y otras razones la comunidad científica -constituida por quienes laboran en una misma área o disciplina- ha introducido sucesivamente en sus modelos de análisis nuevos enfoques provenientes de las ciencias sociales y del mundo intangible de las ciencas humanas, en un esfuerzo por mejorar la capacidad de compresión de la realidad (Kuhn, 2004).

Este proceso, ha hecho que los paradigmas, propios de las diversas disciplinas, hayan sido progresivamente elevados a la categoría de "matríz disciplinar" (Kuhn, 2004), capaces en un momento dado de ofrecer respuestas aceptables mediante un modelo teórico que explica un fenómeno o conjunto de fenómenos dentro de ciertos rangos de verosimilitud y certeza. Sin embargo, progresivamente y en lo sucesivo, dichos modelos y eplicaciones ya no parecen ser suficientes, por una parte, porque el mundo evoluciona y, por otra , porque dicho mundo se complejiza más allá de lo previsto de manera que las disciplinas buscan nuevos modelos y nuevas explicaciones, más coherentes y robustas así como más y mejor autosostenidas por la experiencia.

Es así como las ciencias, que por lo general actúan de conformidad con sus propios paradigmas, se han percatado que ya no alcanzan a explicar a cabalidad la realidad que enfrentan, aún cuando dichas explicaciones hayan estado basadas firmemente en uno o más de sus fundamentos (Easlea, Brian, 1977). Así mismo, ha surgido la percpción entre los eruditos que, a pesar de todo, no todo puede ser explicado con la consistencia esperada reconociendo el hecho que los estimulos a la investigación (Blaug, 1976), por lo general provienen o son inducidos por las anomalías observadas en algún espacio de complejidad, o porque se han identificado dificultades que buscan explicación en instrumentos provenientes del pasado, los cuales, al intentar dar respuesta a las situaciones del presente, parecen no ser capaces de sostener sus postulados originales.
Es así como, muchas veces, los paradigmas tradicionales muestran ciertas insuficiencias al intentar satisfacer interrogantes complejas (Dagum, 1978) del presente, haciendo necesario descubrir nuevas opciones metodológicas que aborden la realidad en toda su multidimensionalidad.

Es justamente esta búsqueda la que genera la denominada revolución científica que, producto de la natural sustitución de paradigmas, sugiere nuevos caminos, nuevas opciones de interpretación y nuevas estructuras conceptuales, las que aún antes de ser cabalmente aceptadas desafían los paradigmas tradicionales presentando nuevas visiones o, a lo menos, nuevas opciones que tal vez ofrezcan nuevas o diferentes capacidades explicativas.

Sin embargo, el proceso no está exento de enfrentamientos, se suscitan luchas entre los partidarios de cada paradigma, unos defienden el anterior y otros promueven la innovación (Barros, 1980). Los primeros intentan dar una explicación relativamente satisfactoria a la anomalía forzando el paradigma tradiconal para hacerlo explicativo. Los segundos promueven la innovación y la creatividad con nuevas explicaciones incorporando nuevas teorías aunque éstas no hayan sido aún reconocidas por la comunidad científica.

Es así como el proceso de cambio científico se desarrolla, incrementa y acumula, a partir de la ciencia normal generalmente aceptada por los iniciados (Popper, 1994), y cuyos postulados fueron progresivamente instalados e integrados a paradigmas tradicionales; pero, el surgimiento de pequeños fenómenos inexplicados, con respecto a los cuales los defensores de la ciencia normal confían en explicarlos sin necesidad de cambiar paradigmas vigentes, ponen en evidencia la tendencia de los expertos a forzar la teoría para incorporar dichos fenómenos, eventualmente inexplicados, ampliando el ambito cubierto por los paradigmas tradicionales.

Se gesta de esta forma la semilla de un enfrentamiento intelectual dando inicio a períodos de confusión y crisis, a la generación de nuevos espacios de innovación y de oposición de modelos hasta que se alcance una re- 
lativa o definitiva integración de nuevos paradigmas que alcancen progresivamente la categoría de aceptados (Easlea, 1977). Con el paso del tiempo y las evidencias, se instala la innovación como tradicional e intenta, desde sus postulados, explicar la realidad, con lo cual se inicia un nuevo ciclo de cambio e innovación (Barros, 1980) de tesis, anti tesis y síntesis a un nuevo nivel de conversación científica, por lo general, de mayor profundidad (Popper, 1994) y, a veces, también de mayor focalización.

Este proceso resulta evidente en el ámbito de las ciencias exactas o naturales, sin embargo, en las ciencias sociales se presentan dificultades y confusiones que complejizan su comprensión. Pero, aún así y de alguna manera, las evoluciones "se introducen en el mundo de las subdesarrolladas ciencias sociales" (Blaug, 1976), proporcionando a los analistas la opción de percibir, analizar e interpretar la realidad social y, con el tiempo, dar origen también a ciclos de confusión, duda, crisis e innovación de los paradigmas tradicionales de investigación social.

En una crítica a Kuhn (Dagum, 1978) se sugiere que el proceso de sustitución de paradigmas implica avanzar desde lo que se denomina "Programas de Investigación Científica", generalmente desarrollados sobre la base de "un núcleo o parte esencial que permanecería invariable" hacia la construcción de nuevos núcleos actualizados y robustos, que por lo general se sostienen de un "cordón de seguridad para el núcleo", constituido por hipótesis auxiliares, mismas que pueden ser sujeto de contrastación, refutación y modificación mediante lo cual se prevén innovaciones, pero que sin embargo, las asumen sin modificar su nucleo central invariable.

De ahí entonces que cuando se destruyen ciertas hipótesis, sin la debida validación e instalación metodológica, se genera una inercia de deslizamiento degenerativo del nucleo, con lo cual se acentúa la confusión (Habermas, 1996). Sin embargo, si las innovaciones conllevan mayor contenido empírico y se cuenta con modelos conceptuales relativamente aceptados, se suscita un deslizamiento progresivo del conocimiento sostenido en las innovaciones, en la medida que las mismas sean capaces de "explicar el éxito previo de su contrario y, al mismo tiempo, lo sustituya por otro que presente mayor poder heurístico" (Dagum,1978).

Es ésta, en esencia, una de las maneras de explicar la génesis del cambio de paradigmas y la evolución de modelos intelectuales de investigación, análisis e interpretación actualizada de la realidad, tanto en el ámbito de las ciencias exactas como de las sociales.

\section{CONSIDERACIONES ÉTICAS}

En general, las implicaciones éticas de las investigaciones académicas son entendidas como el respeto y protección que se da a los sujetos de la investigación. Así por ejemplo, en una investigación clínica, se prevé el resguardo ético pertinente por los eventuales efectos que la investigación pueda tener sobre la integridad de las personas, dado el tipo de trabajo y el tipo investigación que se efectúa sobre ellos.

Sin perjuicio de lo señalado, en el ámbito de las investigaciones sociales desarrolladas sobre la base de estructuras de conocimiento prescrito (Cortina, 2007), el equipo de investigación puede prever efectos éticos menores e incluso inexistente, puesto que, al no tratarse de investigaciones de impacto directo sobre los seres humanos, se deben sólo considerar previsiones de protección en términos de anonimato para con los sujetos objeto de la investigación. Se robustece en consecuencia, la confidencialidad de los datos recogidos así como la objetividad del procesamiento, análisis e interpretación global de ellos. Se destaca, así mismo, los resguardos éticos que dicen relación con la difusión de los resultados de la investigación y sus efectos.

Desde la perspectiva ética de lo público(Echebarría, 2000) se señala que es cada vez más evidente la urgencia de reconocer los efectos de las investigaciones tanto hacia adentro como hacia afuera de las organizaciones y que, en el caso de las entidades gubernamentales, éstas son de naturaleza eminentemente pública (Kliksberg, 1989). Este aspecto adquiere relevancia dado el hecho que la persona y figura del directivo resulta esencial, por una parte, porque interactúa con la comunidad y, por otra, porque propicia la moral que, en virtud de la ética, concierne a las relaciones internas y externas de la organización para con la sociedad a la cual sirve (Cortina, 1994). 
Integrando las visiones enunciadas desde la perspectiva de la ética de un directivo y considerando a su vez el contexto complejo de las instituciones (Echebarría, 200o), se prevé cómo lograr una ética eficaz en la que los miembros de la organización tienden a reconocer sus obligaciones o, a lo menos, su corresponsabilidad en las acciones y en los efectos, en tanto que a su vez reconocen sus derechos en cuanto individuos insertos, en razón de sus trabajos, desempeños y profesiones en una organización pública de suyo compleja (Dejours, 1998). Es por esto que es preciso identificar a los individuos y a las organizaciones sobre la base de una ética fundamental de la cual forman parte, ejerciendo, desde sus respectivas posiciones, un tipo de management que se puede calificar como de tercer tipo.

Se genera así un tipo de management que incorpora atributos como la confianza, la humanización y la calidad de vida, así como la prudencia, la moderación, la flexibilidad y la pro-actividad, generando una serie de conceptos orientadores que revalorizan las organizaciones en sí mismas como esenciales a la vida en sociedad (Pieró, 1986). Por una parte, porque entre sus variables clave está precisamente la conformación de una sociedad sometida a un proceso continuo de aprendizaje y, por otra, por la necesidad de reconocer la importancia de la toma de conciencia ética en el rol permanente que les cabe a los directivos en el ejercicio y análisis de sus propias actividades al interior de sus organizaciones (Frohman, et al., 1976).

\section{ASPECTOS METODOLÓGICOS}

La metodología prevista en este tipo de estudios (Hampson y Morris, 1996) contempla un equipo de investigadores multidisciplinario de áreas tales como la psicología del trabajo, ergonomía, gestión, economía, sociología, entre otras, de manera tal que contribuyan al proceso de análisis desde la perspectiva de los diversos enfoques que representan así como por la diversidad de métodos, opciones de diálogo e interpretación que conllevan para la generación de debates multidimensionales sobre los resultados alcanzados (Kemmis y McTaggart, 1998). De este modo se generan las condiciones necesarias, aunque no exhaustivas, para generar información esencial que permita alcanzar grados superiores de inteligibilidad de la realidad analizada y también incremente y profundice tanto la elaboración de nuevas perspectivas analíticas así como también la mejor interpretación de actividades de naturaleza compleja.

Es así como, sobre la base de lo señalado, el trabajo de campo resulta esencial en tanto y en cuanto ha de ser determinado de común acuerdo entre el equipo interdisciplinario de investigadores, los investigados que progresivamente son incorporados al análisis y las propias instituciones involucradas en el trabajo de análisis e interpretación de la realidad compleja objeto de las indagaciones que el método contemple. Se conforma de esta manera una red multidimensional que representa y enriquece la labor de los equipos de investigación (Barbier, 1996) y muy especialmente la naturaleza de la investigación haciendo de ésta una realidad co-construida a medida que las fases de la misma avanzan en análisis e interpretación.

En lo concreto, el análisis de campo de las actividades en contexto y realizado sobre la base de la observación de una diversidad de prácticas (Barbier y Galatanu, 200o) complejas, conlleva múltiples indagaciones en terreno que pueden incluir desde el análisis de los registros o "huellas", que dejan las actividades desarrolladas, hasta la interpretación multidimensional de acciones, resultados e impactos, mismas que en ocasiones pueden llegar a producir multiplicidad de derivaciones apreciativas de los analistas como de los propios ejecutores de los desempeños analizados.

Es factible también recurrir a medios de soporte tales como documentos, instrumentos y registros de resultados, hasta el hecho de recoger un relato oral o pormenorizado por escrito de las prácticas desarrolladas por los propios sujetos de la investigación (Barbier y Galatanu, $2004(\mathrm{a}))$.

Otra opción es recoger evidencias a través de la realización de seminarios colectivos de análisis e interpretación contando con la participación del equipo de investigación (Hernández et al., 2006)(21), mediante los cuales se generan instancias pertinentes que estimulan la realización de interpretaciones cruzadas entre los diferentes enfoques disciplinarios, enriqueciendo el análisis e inter- 
pretación de las actividades.

Se desarrolla de esta forma un nuevo método de investigación constructivista que, al tenor de lo señalado (Barbier, 1996 ; Hampson y Morris, 1996; Le Boterf. 2001) es el pertinente para catalizar contribuciones de diversas disciplinas tanto en las actividades de recogida, procesamiento, análisis, interpretación y consolidación de datos (Dagum, 1978) como a través del trabajo conjunto y consensuado de diversas disciplinas que contribuyen incluso desde la divergencia que representan.

Por una parte, porque reúne en un único espacio de preocupaciones a diversos mundos profesionales y porque conlleva, eventualmente, al aprendizaje y desarrollo de nuevas competencias (Levy-Leboyer, 1995) vía fertilización cruzada entre los profesionales participantes, los equipos de investigación y los sujetos de la investigación quienes podrían adoptar nuevas y mejores prácticas así como mejores decisiones en la cotidianeidad de sus desempeños profesionales (Garza y Levanthal, 2000).

En lo formativo, este tipo de investigaciones constructivistas y multidisciplinaria, considera la aplicación de tecnologías de indagación, análisis e interpretación complejas que ya han sido probadas en diversas investigaciones (Le Boterf. 2001) y que han proporcionado nuevas oportunidades para identificar, dimensionar y experimentar en torno al aprendizaje de profesionales insertos en sus respectivos contextos de trabajo, proporcionándoles las opciones idóneas para reflexionar e internalizar la eficacia y efectividad de sus desempeños ayudándoles de esa forma a "aprender a aprender" sobre la base de la intelegibilidad y análisis de sus propios desempeños (Barbier, 1996).

\section{ACERCA DEL PROBLEMA DE INVESTIGACIÓN}

Con respecto a los problemas factibles de abordar mediante el método de análisis de actividades (Barbier, 2004), las organizaciones en su diversidad de conformaciones y amplitud de sus propósitos y acciones, pueden ser calificadas como uno de los medios idóneos en los cuales es factible y eventualmente recomendable aplicar este tipo de metodologías, ya sea por el tipo de problemas que surgen así como por la dinámica que és- tas representan.

Es así como se ha reconocido que las organizaciones públicas y privadas se enfrentan a escenarios complejos y multidimensionales (Cortina, 2007; Kliksberg, 1989) en los cuales interactúan con múltiples actores. En ocasiones, se entremezclan con entidades señeras y referenciales del sector de actividad (Beckhard y Reuben, 1988), por una parte, porque son ellas las que enfrentan los cambios fundamentales que impactan a la sociedad y, por otra, porque son capaces de internalizar las evoluciones que experimenta la sociedad en su conjunto incorporando progresivamente complejidades a su campo de actuación (Habermas, 1996).

Desde otra perspectiva, las organizaciones hacen parte de sectores cuya dinámica puede ser lenta o acelerada, así como también evidenciar trazas de volatilidad profunda o inexistente entrecruzándose mutuamente y haciéndose cargo de los espacios de complejidad que la propia sociedad les muestra (Argyris y Schon, 1983) en un proceso que estimula el origen y creación de organizaciones con capacidad de constituirse en referentes y cuyos procesos de transformación impactan directamente la globalidad anulando, a veces, la capacidad de reacción y respuesta de otras menos estructuradas o menos veloces.

Es así como la propia naturaleza de las organizaciones les otorga las capacidades de enfrentar y asumir la complejidad social lo que resulta ser de vital importancia y especialmente relevante por el rol que eventualmente les cabe jugar en la articulación de redes complejas como las que cada industria representa (Barbier J-M , Galatanu O. 2004 (b)).

Es, en este contexto de cambio, que es preciso disponer de un método de abordaje de la realidad compleja mediante la creación de nuevos espacios de conversación y de co-construcción (Barbier, 2004 (a); Bruner, 1991) que conlleve la generación de un marco de reflexión y análisis para producir nuevos modelos mentales (Barbier y Galatanu, 2004 (a)), nuevos paradigmas de actuación y de investigación respecto del rol que le cabe a las organizaciones y que propicie grados superiores de inteligibilidad en quienes laboran o las dirigen (Barbier 1999 (a)), considerando que todas las actividades se insertan 
en un proceso de implementación y puesta en práctica en permanente evolución.

El escenario descrito en los párrafos anteriores permite vislumbrar la necesidad de que existan mecanismos pertinentes que permitan describir de manera sistemática - en proximidad o a distancia - las actividades en las organizaciones (Barbier, 2004. (a) ; Bruner, 1991), así como reflexionar acerca de las actividades que les son propias.

De esta forma, el análisis y comprensión de un determinado acto hace que éste sea lo que le corresponde ser de acuerdo con una determinada prescripción que lo define como una actividad socialmente validada (Barbier y Galatanu, 200o; Barbier y Galatanu, 2004 (a)). Porque la actividad conjuga la participación de diversos actores clave que se desempeñan de acuerdo con sus propios modelos mentales y porque el autoanálisis e interpretación de sus propias actividades les permiten la innovación y cambio (Barbier, 1999 (b); Benson y Hughes, 1983) de sus actuales desempeños; en un asunto que puede ser abordado mediante el análisis transversal de los desempeños propiciando el cruce de interpretaciones provenientes de escenarios diversos, dinámicos y complejos.

\section{ACERCA DE LAS HIPÓTESIS DE INVESTIGACIÓN}

Con todo, la complejidad de las actividades conlleva la necesidad de emplear múltiples opciones de investigación sustentadas en hipótesis que es menester circunscribir a sus dimensiones más relevantes (Belisle y Linard, 1996). Por una parte, el análisis de actividades se enfoca precisamente en la toma de conciencia y reflexión subrayando la importancia del análisis fino de las acciones en un escenario complejo. Además, no hay duda de que son los propios individuos quienes deben identificar las competencias necesarias para enfrentar las nuevas realidades (Beckhard y Reuben, 1988; Hammersley y Atkinson, 1983) además de reconocer la existencia de actores claves influyentes tanto por el ejercicio profesional como por las jerarquías de autoridad que ejercen en los diversos niveles en que se desenvuelvan.

Estos actores diversos representan instituciones y colectivos que es preciso relevan tales como colegios profesionales, organizaciones sindicales, autoridades locales, dirigentes vecinales, pares y subordinados, entre otros. Actores que interactúan en escenarios complejos con colectivos dispersos compuestos por individuos quienes, de una o de otra forma, definen una cierta autopercepción de las actividades así como valoran su actuación e interacción con contrapartes personales e institucionales poniendo de manifiesto conductas y competencias identificables mismas que materializan el hecho de "aprender a aprender" a través de la acción (Barbier, 2000 (a); Barbier, 1996).

Es así como se puede apreciar opciones hipotéticas (Barbier, 1996) que pueden ser explicitadas: A modo de ejemplo, en la siguiente expresión: H1: Que la naturaleza de las actividades individuales en sus contextos de trabajo "condicionan las competencias cognitivas y emocionales de la persona para actuar" o bien, H2: Que la naturaleza de las actividades individuales en sus contextos de trabajo "no condicionan las competencias cognitivas y emocionales de la persona para actuar", generando un tipo de análisis que se puede realizar a) sobre la base del tiempo institucional en articulación con el espacio profesional, b) a partir del espacio privado del individuo estableciendo distinciones entre el trabajo real y el trabajo prescrito y c) que las acciones de la persona son realizadas en nombre del Estado, en nombre del Establecimiento o en nombre de Valores a los cuales adhiere (Le Boterf, 2001) entre otras opciones de análisis de las actividades.

Cabe mencionar que, desde la perspectiva del enfoque dinámico de investigación en el contexto de las actividades y prácticas individuales, definir hipótesis al inicio del proceso de indagación puede dar como resultado hipótesis no originales y hasta no trascendentes (Barbier J.M. 1999 (b)).

Es por ello que, desde la dinámica de las investigaciones constructivistas, es perfectamente posible y hasta esperable que en su propio desarrollo se vayan generando nuevas hipótesis, que tiendan a ser más precisas y al mismo tiempo más originales (Barbier, 1996), entre otras razones, porque las hipótesis del análisis de prácticas y de actividades se definen durante y después pero casi nunca antes de la investigación, en consecuencia, es necesario observar, reconocer y valorar la polifuncionalidad de los actos así como su relación con el contexto en el cual 
se desempeñan. Es preciso reconocer así mismo la dinámica de cambio del escenario complejo que han de enfrentar tanto adentro como fuera de la organización y la multiplicidad de actos y actores involucrados, entre otros factores condicionantes de las actividades analizadas.

\section{ACERCA DE LOS OBJETIVOS DE INVESTIGACIÓN}

Respecto de los objetivos de investigación en estudios sujetos a la dinámica del constructivismo (Levy-Leboyer, 1995), es preciso reconocer algunas consideraciones esenciales, en primer lugar, con relación a los escenarios que pueden presentarse, por un lado, en estado de pasividad y estancamiento y, por otro, evidenciando situaciones de turbulencia y volatilidad motivados en causales múltiples, eventualmente, por el impacto de procesos de cambio hacia los cuales deriva la sociedad (Covarrubias, 1998).

Así mismo, es menester reconocer la influencia que resulta de la implementación de reformas capaces de generar desafíos y espacios de oportunidades a las personas y a las organizaciones (Covarrubias, 1998), tal vez, por las múltiples interacciones que ocurren entre mundos profesionales diversos, y porque la gestión de procesos de cambio requiere de la incorporación del recursos humanos cada vez más competentes, a quienes se los define como el factor clave que sostiene y profundiza las innovaciones y dentro de los cuales, los directivos, son precisamente valorados como esenciales por el rol de liderazgo que les corresponde realizar especialmente en las fases de implementación de las innovaciones (Belisle y Linard, 1996).

Es así como el objetivo general, habitualmente declarado de manera genérica en estas investigaciones, busca establecer un marco de reflexión, análisis e interpretación de la realidad desde la perspectiva de la complejidad para producir nuevos modelos mentales (Hammersley y Atkinson, 1983), mismos que se evidencian en los individuos insertos en una determinada organización, comenzando con las actividades propias de la persona así como también con las que derivan de las evoluciones de las organizaciones como un todo considerando que éstas también se encuentran insertas en un contexto en plena evolución a causa de transformaciones y adaptaciones que experimenta el mundo de las organizaciones, sean éstas públicas o privadas (Kliksberg, 1989).

\section{EL TRABAJO DE CAMPO}

Para lograr propósitos de investigación, como los descritos en los párrafos anteriores, se ha optado habitualmente por el desarrollo secuencial de pasos propios de la investigación constructivista (Covarrubias, 1995 (a); Bruner, 1986), a decir de la secuencia que comienza con algunos cuestionamientos que luego se problematizan definiendo tanto, un nivel de análisis, como líneas de investigación que orientan el acopio de datos, en una primera fase que estimula, en consecuencia, la aplicación de una cierta metodología e través de una secuencia de momentos de indagación sostenidos en una cierta epistemología asociada a preguntas de investigación que se traducen en objetivos que evolucionan desde lo general hasta lo específico y que habrán de ser desarrollados mediante la utilización de una metodología determinada.

Desde el punto de vista descrito (Covarrubias, 1995 (b); Covarrubias, 1999), es menester precisar objetivos tales como: a) caracterizar el contexto institucional en el que se desempeñan los individuos, b) caracterizar la unidad orgánica en la cual las personas ejecutan sus actividades habituales y c) establecer los mecanismos pertinentes de observación y análisis de registros referenciales de las realidades analizadas.

Desde la perspectiva de estudios individuales (Wertsch, 1988) es preciso: a) identificar el desempeño de las actividades de las personas y sus evoluciones $y, b)$ obtener una representación de dichos individuos en sus actividades cotidianas.

Desde el punto de vista del desempeño colectivo (Martínez, 1995), es necesario: a) describir en proximidad las actividades habituales, b) describir a distancia el conjunto de actividades de las personas.

Finalmente, y volviendo al análisis individual de las actividades (Hampson y Morris, 1996) (16), cobra sentido: a) identificar los actos de interacción de las personas con sus pares y b) generar los modelos mentales que sostienen las actividades de las personas en sus respectivos contextos de cambio (Glasersfeld, 1993)(44). 
La secuencia de objetivos señalados se materializan en acciones de indagación sin requerir necesariamente de algún modelo previo que oriente la búsqueda de manera normativa (Martínez, 1998) sino que más bien, el propósito es precisamente indagar la realidad para derivar de la misma los antecedentes que permitan modelarla como un hecho positivo.

Es así como la serie de objetivos de una investigación desarrollada sobre la base de un modelo constructivista (Pourtois, et al., 1992), se realiza progresivamente partiendo desde los análisis macro estructurales hasta derivar en los estudios individuales y grupales que se requieran de conformidad con el objetivo general de la investigación.

\section{SINTETIZANDO UNA METODOLOGÍA PARA LA INVESTIGACIÓN}

La investigación del tipo análisis de actividades y prácticas, que se ubica entre los estudios cualitativos constructivistas, abre nuevos espacios de indagación de realidades complejas (Sanguino, 2003), en su génesis con el propósito de lograr grados superiores de "inteligibilidad" de las actividades y como medio para "aprender a aprender" a partir del análisis y razonamiento del desempeño propio (Barbier, 2000 (a)).

Los procedimientos de recogida de datos dan lugar a variados procesos de observación y análisis, abordando desde la perspectiva de una investigación cualitativa, los componentes del desempeño de manera de construir un campo interdisciplinario de análisis $y$, en algunas ocasiones, contra disciplinario de compresión de la realidad (Siegel y Castellán, 1985).

Este tipo de estudios atraviesa las humanidades, las ciencias sociales y la física (Covarrubias, 1995 (b)) y apunta a la profesionalización, a lo menos, en dos sentidos, primero, la profesionalización de las actividades en el sentido de organización social y, segundo, la profesionalización de los actores -aprender a aprender- en el doble sentido de transmisión / producción de saberes y competencias (Barbier, 2000 (a)) que generen identidad profesional (Barbier y Galatanu, 2000).

El universo objetivo de este tipo de investigaciones se conforma, en general, por los individuos localizados en su propia área de desempeño ya sea en el contexto de organizaciones públicas como privadas (Le Boterf, 2001). Además, como es sabido, las poblaciones sujeto de este tipo de trabajos de investigación (Covarrubias, 1995 (a)) suele ser variable de acuerdo con el tamaño sectorial, la dinámica de los cambios involucrados y las transformaciones derivadas de la propia complejidad de sus procesos e interrelaciones con el entorno global.

Respecto de la población y muestra, este tipo de investigaciones sugiere determinar muestras por conveniencia, considerando la mejor representatividad de individuos, tipo de establecimiento e institución, complejidad de operaciones así como diversidad de contextos en los cuales los individuos indagados se insertan (Pourtois, et al., 1992). El número de elementos va de 5 a 10 provenientes de las instituciones participantes.

El marco de elementos de muestra elegibles para este tipo de trabajos (Martínez, 1998), considera estudios de campo de cobertura focalizada, por lo general, constituido por personal operativo, profesionales, gestores de primer y segundo nivel así como directivos de nivel superior incluidos propietarios, todos los cuales conforman un conglomerado factible de ser seleccionados en consideración a la diversidad de sus desempeños así como por su capacidad de gobierno y de sus implicaciones tanto hacia adentro como hacia fuera de la organización, según corresponda.

Desde una perspectiva interinstitucional, la muestra puede considerar elementos representativos de las diversas realidades organizacionales y territoriales seleccionados por conveniencia (Landier, 1992). El número de unidades puede fluctuar entre un mínimo de 5 y un máximo de 10 directivos por segmento analizado, pudiendo totalizar entre 25 y 50 de conformidad con el tamaño del universo global y de la cobertura que se quiera dar al estudio.

Entre las técnicas de recolección de datos, se ha previsto, que en las investigaciones de análisis de actividades así como en el estudio de fenómenos sociales, se recurra a identificar, por medio de auto observación y luego mediante observación en cercanía y directa, 
los aspectos relevantes de la actividad analizada (Siegel y Castellán, 1985). Así también se puede hacer uso de técnicas de acompañamiento y verificación de registros de evidencias del desempeño así como el análisis de discurso oral y escrito de los individuos sujetos de la indagación, entre otros métodos.

La investigación se puede centrar, en general, en el estudio de los métodos o estrategias empleados por las personas para construir, dar sentido y significado a sus prácticas sociales cotidianas. Las cuestiones de investigación se enfocan, en consecuencia, en la interacción verbal y el diálogo. En tanto que, entre los métodos habituales se puede considerar el análisis de discurso, cuyas fuentes derivan de la semiótica y sus técnicas se expresan en el diálogo y en el registro constituyendo medios de soporte diversos tales como, texto, audio y video, además de notas de campo, entre otros (Belisle y Linard, 1996).

Como una forma de ejemplificar la puesta en marcha de una investigación sobre la base del método constructivista que deriva del análisis de actividades, la Tabla 1 muestra una serie de 7 objetivos factibles de ser investigados y las correspondientes 13 actividades que se sugieren para su logro.

\begin{tabular}{|c|c|}
\hline $\begin{array}{l}\text { Objetivo } \\
\text { específico } 1\end{array}$ & Caracterizar el contexto institucional en el que se desempeñan los directivos. \\
\hline Actividades & $\begin{array}{l}\text { 1. Definición del contexto o marco de trabajo que se describe y analiza en el curso de la } \\
\text { investigación, considerando el contexto general, contexto institucional y el marco de la } \\
\text { actividad, el tiempo, los espacios, los modos de interacción, el reconocimiento y las zonas de } \\
\text { turbulencia, entre otros antecedentes. }\end{array}$ \\
\hline $\begin{array}{l}\text { Objetivo } \\
\text { específico } 2\end{array}$ & Identificar el desempeño de las actividades de los directivos y sus evoluciones. \\
\hline Actividades & $\begin{array}{l}\text { 2. Observación directa de actividades de gestión en red: comprende una descripción del } \\
\text { contexto de trabajo y organizacional; } \\
\text { 3. Recolección de huellas, durante a lo menos una semana, determinación de la duración y } \\
\text { cronograma real de las actividades y tipos de intervenciones del directivo, sean éstas de } \\
\text { análisis, evaluación, sugerencia, persuasión, arbitraje, decisión, entre otras. } \\
\text { 4. Determinación del recorrido del directivo mediante la identificación de su experiencia } \\
\text { profesional, recorrido de formación inicial y continua; } \\
5 \text {. Elección de la carrera directiva y modo de entrada en la función de directivo, contexto de la } \\
\text { historia personal, entre otros factores. }\end{array}$ \\
\hline $\begin{array}{l}\text { Objetivo } \\
\text { específico } 3\end{array}$ & $\begin{array}{l}\text { Obtener una representación de los directivos en cuanto al desarrollo de sus actividades como } \\
\text { gestores de red. }\end{array}$ \\
\hline Actividades & $\begin{array}{l}\text { 6. Análisis de discurso (procedimiento oral y escrito) sobre las actividades; mediante el cual } \\
\text { se procede al análisis de las declaraciones que formula el directivo; } \\
\text { 7. Determinación de la duración y cronograma real de las actividades y tipos de intervenciones } \\
\text { del directivo, sean éstas de análisis, evaluación, sugerencia, persuasión, arbitraje, decisión, } \\
\text { entre otras. } \\
\text { 8. Establecimiento de la significación social y la significación laboral de la actividad directiva. }\end{array}$ \\
\hline $\begin{array}{l}\text { Objetivo } \\
\text { específico } 4\end{array}$ & Describir en proximidad las actividades de los directivos. \\
\hline Actividades & $\begin{array}{l}\text { 9. Definición de estructuras, distintivos de simbolización, reflexión y de singularización de la } \\
\text { actividad directiva de los gestores de la red asistencial. }\end{array}$ \\
\hline $\begin{array}{l}\text { Objetivo } \\
\text { específico } 5\end{array}$ & Describir a distancia el conjunto de actividades de los directivos. \\
\hline Actividades & $\begin{array}{l}\text { 10. Comparación con las actividades prescritas, que se inducen de la descripción de puestos, } \\
\text { de normas, reglamentos, definiciones de perfiles procedentes de una reforma etc. y por } \\
\text { comparación con las actividades prescritas precedentes a la reforma, se establece el marco o } \\
\text { contexto que configura las actividades estudiadas, los actores involucrados en esta } \\
\text { configuración, la descripción de las actividades de los actores y sus interacciones lo cual } \\
\text { implica un cruzamiento de las observaciones y del discurso para acercarse a una } \\
\text { interpretación de la actividad. }\end{array}$ \\
\hline $\begin{array}{l}\text { Objetivo } \\
\text { específico } 6\end{array}$ & Identificar los actos de dirección de los directivos. \\
\hline Actividades & $\begin{array}{l}\text { 11. Determinación de los problemas planteados en el terreno, por ejemplo mediante el análisis } \\
\text { de "viñetas" (observaciones incluso parciales) que provienen del terreno; } \\
\text { 12. Procesamiento multi disciplinario de datos. }\end{array}$ \\
\hline $\begin{array}{l}\text { Objetivo } \\
\text { específico } 7\end{array}$ & $\begin{array}{l}\text { Generar los modelos mentales de los gestores respecto de las actividades directivas en el } \\
\text { contexto de los cambios sectoriales que enfrentan. }\end{array}$ \\
\hline Actividades & 13. Interpretación, análisis y modelamiento multidisciplinario de datos. \\
\hline
\end{tabular}




\section{TÉCNICAS DE RECOGIDA, ANÁLISIS Y EVALUACIÓN DE} RESULTADOS

El análisis de actividades, la metodología de gestión de cambios y la Investigación Acción (Bruner, 1991), se conciben desde una concepción positivista de la investigación. Se sostiene en la relación investigador/investigado por medio de la cual se forja un nuevo tipo de investigador que realiza su trabajo de forma sistemática a través de un método reflexivo y orientado a interpretar la realidad en sus diversas dimensiones. Las cuestiones de investigación dicen relación con la obtención de mejoras, en este caso, de competencias profesionales así como también respecto de los eventuales cambios sociales que se deban enfrentar y las consecuentes actividades de interacción entre los actores organizacionales.

El método contempla etapas recursivas a decir, de investigación - acción sostenida en la teoría crítica acompañada de técnicas misceláneas por su diversidad pero plenamente integradas en el método constructivista que propone este documento (Barbier, 1996 ; Bruner, 1991). Establece además, diversas pautas en torno a la documentación, observación, intervención, entrevistas, recolección de datos y análisis (Covarrubias, 1995(a)).

Se prevé así mismo, su recursividad en períodos que van desde a lo menos 2 y como máximo 5 días de acompañamiento en el lugar de trabajo en el cual se lleva a efecto la observación directa e inmediata del individuo en terreno, habida tenencia de las autorizaciones de acceso a los niveles de desempeño en los cuales se estructuran las organizaciones involucradas (Argyris y Schon, 1983).

Por otra parte, dada la naturaleza de las investigaciones de análisis de actividades y prácticas (Wertsch, 1988), se considera en general adecuado producir datos relacionados con la descripción o reconstrucción analítica de carácter interpretativo de la realidad en sus diversos contextos (Bruner, 1986), así como el levantamiento de la cotidianeidad que deriva de las formas de vida y estilos de trabajo (Pourtois, et al., 1992) en la organización y en sus relaciones con otras entidades y personas. Del mismo modo es necesario analizar las estructuras organizacionales y las conformaciones sociales del grupo investigado, entre otros temas claves asociados a la etnografía y psicología de las organizaciones (Frohman y Kavanagh, 1976; Schön, 1983).

Las interrogantes de Investigación (Le Boterf, 2001), en casos como los señalados dicen relación con el levantamiento de hechos, descripción e interpretación de valores, síntesis de ideas fuerza, relato de prácticas de los grupos humanos a partir de un método dialógico y multidimensional de factores concatenados de manera de logar la mejor interpretación de situaciones propias de la antropología y sociología de organizaciones complejas (Lincoln y Denzin, 1994).

Las técnicas conllevan entrevistas no estructuradas además de observaciones de campo del investigador. Se prevé el registro de notas así como la identificación de huellas dejadas por el individuo tales como documentos, registros, diagrama de redes sociales que derivan y son propios del ejercicio de su labor, entre otros (Hanna, 1990).

Entre los programas computacionales para el análisis de datos se identifican a) programa Aquad (Análisis of Qualitative Data) que incorpora seis componentes, a decir: Parámetros globales, textos y apuntes, códigos, hipótesis, apéndice y minimalización; b) programa NUDIST (Non-Mumerical Unstructured Data Indexing, Search and Theorizing) cuya base del trabajo son los sistemas de documentos y sistemas de indización y c) el que otorga la Licence $\mathrm{D}^{\prime}$ utilisation pour una solution ADT.Alceste; programa cuya Version Reseau Local está disponible Pour Windows 98; Me 2000 et XP. Con recursos Logiciels: Module Alceste Limite A 1 Mo Pour L'anályse de Texte Editeur de Texte EdImage: Navigateaur de Consultation Des Resultats Rapport Resumé; Module Graphique CDH. CAH. AFC.

Las técnicas de análisis de datos (Covarrubias, 1995 (a)) así como la evaluación de resultados implica el procesamiento de datos cualitativos mediante actividades 
como la reducción de datos, la separación de unidades, la síntesis y agrupamiento e identificación y la clasificación de elementos (Le Boterf, 2001). Además se considera la disposición y transformación de datos mediante para contribuir a un proceso de obtención y verificación de conclusiones.

Con relación a los procedimientos, se sugiere la constitución de un equipo coordinador de la investigación encargado de vincular los equipos de investigación con las organizaciones públicas o privadas así como con los respectivos directivos sujetos de la investigación en terreno (Pourtois, et al., 1992). Dicho equipo coordinador se habrá de ocupar también de la programación, duración y cronograma de las actividades de investigación y tipos de intervenciones de campo dentro de los plazos previstos. Los análisis de contexto pueden ser definidos por equipos zonales relacionados con las zonas y establecimientos objeto de la investigación como se muestra en la figura 2.

Figura 2 Un método para los trabajos de campo

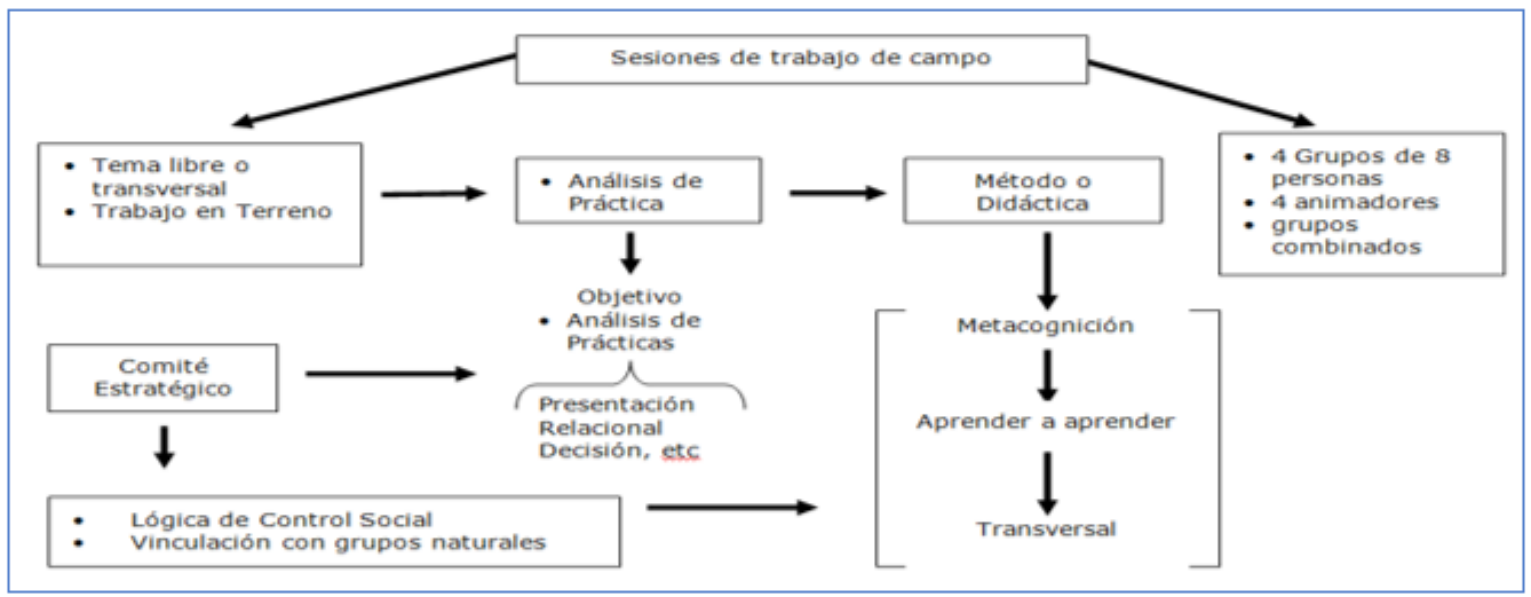

FUENTE: ELABORADO SOBRE LA BASE DE COVARRUBIAS VILLA, FRANCISCO. 1998; DAGUM, C. 1978; BARBIER J.M. 1996

La recomendación de crear un "equipo coordinador central de la investigación”, tiene entre sus propósitos distinguir las funciones propias de cada zona como también determinar las opciones metodológicas más adecuadas de integración y coordinación de acciones para este tipo de indagaciones (Covarrubias, 1995 (a)) y asegurar la consistencia y coherencia de los datos recogidos para su posterior procesamiento (Barbier, 1996). Podrían consi- derar además las funciones de definición de los tiempos mínimo de presencia en terreno de los equipos de investigación con y sin citas organizadas para entrevistas, la coordinación de la auto percepción y percepción por parte de terceros a distancia y en cercanía de las actividades investigadas, además de asegurar las opciones para acceder a actividades complejas tal como ocurren en su respectivo contexto de desempeño (Figura 3).

Figura 3 Estructura de coordinación de investigación

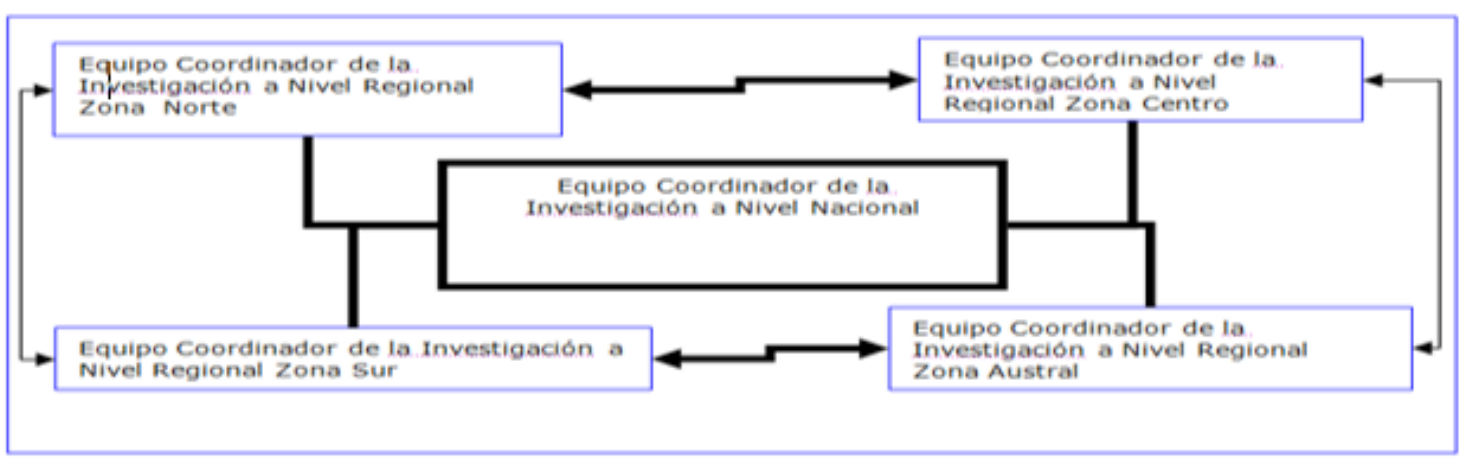

FUENTE: ELABORADO SOBRE LA BASE DE COVARRUBIAS VILLA, FRANCISCO. 1998; DAGUM, C. 1978; BARBIER J.M. 1996. 
El equipo coordinador tendría que gestionar la estimación de los tiempos de investigación adicionales para asegurar disponibilidades especificas, por ejemplo, accesos a terreno fuera del tiempo formal de trabajo y podría expandir el tiempo de investigación para efectos de asegurar la fase de maduración del análisis e interpretación de los datos (Landier, 1992).

Con relación al equipo de investigadores de campo, se sugiere que éste sea constituido por investigadores provenientes de disciplinas distintas (Edvinnson, et al., 200o) y que cada uno de ellos constituya un grupo de trabajo a su cargo estableciendo vínculos de co-investigación con las redes locales en cada zona geográfica.

\section{ACERCA DE LOS RESULTADOS ESPERADOS}

En general, este tipo de investigaciones prevé resultados con impacto a lo menos en dos ámbitos que naturalmente se subordinan a la ética en las organizaciones conforme criterios de responsabilidad social (Dejours, 1998) y que se hacen efectivos a nivel de las personas y a nivel de las organizaciones (Argyris y Schon, 1983).

Entre los resultados de primer orden o de "impacto individual" (Barbier y Galatanu, 2004 (b)) se menciona que este tipo de investigaciones permite poner en evidencia factores claves que definen las exigencias al sector de actividad de las organizaciones. Por otra parte, tienden a igualar las exigencias del mundo privado respecto del público, incorporando términos tales como eficiencia, productividad y satisfacción de clientes, entre otros.

El impacto a nivel de las personas (Barbier, 2004 (a)) contribuye a racionalizar la natural evocación de un modelo y lenguajes tradicionales de las organizaciones privadas que deben compatibilizarse y contextualizarse con el actual escenario orientado hacia el incremento del capital intelectual (Limone y Cademártori, 1998) de las organizaciones públicas. Este tipo de evoluciones organizacionales estimulan sucesivamente la reforma y los cambios, por una parte, con respecto a un nuevo modelo de gestión y, por otra, con relación a la gestión pública asimiladas cada vez más a los criterios y conceptos del sector privado (Kliksberg, 1989).

Es así como el análisis de actividades paulatinamente se inserta en un nuevo modelo de gestión en el que la auto gestión de organizaciones es la base, permite profundizar un proceso de profesionalización de la función de las personas en todos sus nodos y permite a su vez la identificación y transversalidad de buenas prácticas generando aprendizaje, capacidad homeostática y autopoiesis organizacional (Johnson-Laird, 1983) en un proceso constante de aprender a aprender de los individuos en sus respectivas organizaciones (Barbier, $2000(a))$.

Además, las sucesivas fases de investigación que se consideran fundamentales en el análisis de actividades, permiten progresivamente la construcción de un modelo mental explícito de las actividades, la racionalización y objetivación de las prácticas, y en definitiva, la "inteligibilidad" de las actividades por parte de los propios sujetos de la investigación. Este proceso se traduce en declaraciones verbalizadas, en discursos interpretativos orales o escritos que propician la toma de conciencia de las actividades, la racionalización del contexto que rodea esas prácticas, la identificación y caracterización de los actores involucrados y la identificación de las motivaciones que las activan.

Se gesta en consecuencia la inteligibilidad asociada a un proceso de racionalización de la profesionalización en sus dos sentidos, primero, de la organización social y, segundo, de la transmisión y producción de saberes para el sostenimiento de competencias complejas (Barbier, 1999(a)).

Se genera además un marco de reflexión, nuevos modelos mentales (Senge, 1995; Spencer, 1993) y una progresiva aproximación a la toma de conciencia de las prácticas incorporando paulatinamente la cuestión ética en sus acciones y decisiones (Echebarría, 2000). Facilita la racionalización y mejoramiento continuos de las actividades humanas, así como también contribuye a la identificación de problemas directivos y a la obtención de soluciones consistentes y coherentes con 
el propósito de viabilizar nuevas capacidades de gestión producto de la contextualización y re contextualización de los aprendizajes alcanzados.

Entre los resultados de "impacto organizacional" se destaca que los hallazgos permiten incrementar los grados de calidad en su más amplio sentido (Jackson y Ashton, 1998) y, a nivel de directivos, contribuye a facilitar el proceso de tránsito de los antiguos sistemas de gestión a los nuevos sistemas capaces de incorporar las reformas sectoriales respectivas y la consecuente complejidad de desempeños en evidente y paulatino incremento (Le Boterf, 2001).

En la medida que esta investigación coincide con la fase de implementación de la reforma que viven los diversos sectores tanto públicos como privados del país, se superan resistencias, se generan nuevas competencias en los grupos humanos y equipos de trabajo, se hacen realidad prácticas exitosas y también se identifican falencias que terminan por generar nuevos modelos mentales que evidencian e instalan aprendizajes tanto individuales como organizacionales (Johnson-Laird, 1983).

Se genera en consecuencia, un proceso donde se hacen efectivos factores de aprendizaje individual y organizacional que son auto determinados por los propios sujetos de la investigación (Barbier, 1999 (b)). Se produce a sí mismo, un nivel superior de autoaprendizaje (Barbier, 2000 (a)) y desarrollo de competencias que ayudan a los profesionales a ejercer y evaluar la eficacia de sus propias actividades y los efectos que éstas provocan en los demás. Del mismo modo facilita la comprensión de los mecanismos que activan el desempeño individual y que explican las motivaciones personales, aclara las disfunciones organizacionales (Frohman y Kavanagh, 1976) y permite identificar, hacer conciencia y mejorar las prácticas (21). Facilita la internalización de nuevas herramientas que hayan demostrado ser exitosas, tanto para definir competencias como para mejorar los procesos de formación y, desde ahí, impactar en el mejoramiento de las actividades en sus espacios de desempeño (Johnson-Laird, 1983).

Finalmente, el método de análisis de actividades y prácticas hace factible el ejercicio de recursos personales (Hammersley y Atkinson, 1983) para enfrentar un entorno complejo. Genera nuevos escenarios de aprendizaje consistente con la experiencia de individuos que interactúan al interior de las organizaciones y que son parte constitutiva de la complejidad que enfrentan adaptándose sucesivamente a un entorno cambiante. Desarrolla en las personas elementos resiliencia (Frohman y Kavanagh, 1976) que les permiten asumir los cambios que experimentan micro entornos cada vez más complejos y que pueden sucesivamente ser más eficaces a los propósitos de las organizaciones. Se establecen de esta forma las bases, las implicaciones del aprendizaje individual y colectivo (Piaget, 1975/1983) así como las redes culturales de mejoramiento continuo de las organizaciones tanto del sector público como del privado que hacen suyo los nuevos desafíos y evolucionan con naturalidad conforme a ellos.

\section{APLICABILIDAD DE LOS RESULTADOS}

En general el impacto previsto por investigaciones del tipo análisis de actividades y prácticas como la que en estas páginas se describe, se asocia más a cambios de tipo cualitativo que cuantitativo (Le Boterf. 1998), puesto que, en este tipo de estudios es difícil pensar en indicadores cuantitativos inmediatos, sino que más bien, es posible pensar en efectos de mediano o largo plazo de impactos cualitativos, tales como nuevos comportamientos e innovadas concepciones de la realidad (Johnson-Laird, 1983) que propician grados superiores de comprensión e inteligibilidad superior de las actividades humanas, de sus implicaciones y efectos en los demás y en el sistema del cual forman parte (Johnson-Laird, 1990; Piaget, 1975/1983).

Entre los impactos cualitativos esperados es posible mencionar la formación por competencias, dado que los resultados esperados constituyen en sí mismos elementos claves de una estrategia de formación por competencias, que combina 
la formación con la reflexión y la investigación en el lugar de trabajo (Le Boterf, 2001). Reconoce el valor de este último aspecto como espacio de aprendizaje, además permite contribuir a la resolución de problemas de la práctica (Barbier y Galatanu, 2004 (b)) y disminuye las brechas de competencia de las personas para asumir nuevos trabajos en contextos de complejidad (Cortina, 2007), de modo que, la experiencia, el análisis y la interpretación interdisciplinaria de la realidad (Edvinnson y Malone, 2000) facilitan el tránsito a un nuevo modelo de comprensión de la realidad (French, 1969).

En general, se prevé un proceso de incorporación de nuevas prácticas que paulatinamente viabilicen los cambios implícitos en las transformaciones que experimenta la sociedad y sus organizaciones (French y Bell, 1990). Concierne al desarrollo y funcionamiento del trabajo en equipo (Denison, 1991) y otorga al análisis de actividades la capacidad de coordinar y potenciar acciones con eficacia, contribuyendo a generar relaciones simétricas así como también generar un diálogo eficaz y constructivo entre los actores organizacionales.

El método permite potenciar el desarrollo de competencias capaces de articular, integrar y producir nuevos aprendizajes organizacionales en un escenario de interacción que establece las bases del aprendizaje colectivo (Piaget, 1975/1983).

Finalmente, se propicia el establecimiento de nuevos factores de cambio cultural (Denison, 1991) en atención a la dinámica del cambio mismo y a la complejidad de la implementación, de modo que es factible que pueda ser redefinida a partir de nuevas metas de aprendizaje (Hammersley y Atkinson, 1983).

\section{DE LA PROTECCIÓN DE LOS RESULTADOS}

Dado el tipo de investigaciones que caben dentro del modelo descrito en estas páginas, se prevé a lo menos, dos tipos de resguardos de protección de los resultados.

Primero, la protección de la confidencia- lidad de los datos individuales recopilados de los directivos sujetos de la investigación (Sanguino, 2003), en función de lo cual, se sugiere que los equipos investigadores se comprometan a no difundir públicamente elementos individuales recogidos por observación directa, de las entrevistas o de la recopilación de huellas, salvo bajo el velo del "anonimato" de los grandes números o de la reserva de la identidad si es que es preciso presentar un caso individual (Belisle y Linard, 1996). En general, se espera que los equipos de investigación se comprometan a publicar o presentar en reuniones, sólo datos colectivos o casos anónimos.

Segundo, la protección intelectual de los resultados producidos por un investigador o un equipo de investigadores tiene a lo menos que cumplir con el resguardo de la propiedad intelectual en consideración a que el dominio sobre los bienes intelectuales, o de propiedad, son la base sobre la cual se asienta el progreso de la academia. La noción o naturaleza de este dominio es determinante por cuanto "Nadie es dueño de una cosa, a no ser que esté en su potestad usarla...". De esta manera el dominio sobre los bienes intelectuales implica la opción voluntaria del no dominio (De Soto, 1975) en razón de lo cual el producto generado puede ser transferido a otros y por su intermedio a la sociedad como un todo.

Por otra parte, la eventual retro información organizacional hacia los propios sujetos de la investigación (Barbier y Galatanu, 2004 (b)) como usuarios principales, recibirán los resultados parciales y totales de la investigación con las conclusiones correspondientes a sus respectivos niveles de análisis y aplicabilidad.

Por otra parte, en general, la difusión y publicación de resultados parciales implica que cada equipo de investigación, en su dominio regional o zonal según corresponda, podría adoptar la decisión de publicar los resultados de su trabajo, así como también transferir resultados parciales a su contraparte regional e internacional. Sin perjuicio de lo cual, la difusión y publicación internacional es también posible en la medida que se 
cuente con la opción de que los resultados pudieran también ser incorporados en una publicación a dicho nivel.

\section{CONCLUSIONES}

El presente trabajo analizó de manera sistemática la relación que existe entre la técnica del análisis de actividades con los métodos de investigación cualitativa. Se describieron las dimensiones que dan al análisis de actividades el carácter de método de indagación científica de la realidad y que le permiten alcanzar el carácter método, en tanto y en cuanto, en la investigación cualitativa, el análisis de actividades es pertinente para la identificación, análisis y consolidación de nuevas prácticas de investigación.

Los conceptos utilizados en el presente trabajo permiten fundamentar algunos cambios epistemológicos que se ubican a la base de los métodos de investigación cualitativa, por cuanto conlleva la innovación en los procesos de investigación, especialmente cuando estos poseen $\mathrm{ca}-$ racterísticas complejas.

El análisis realizado a lo largo del presente trabajo permite así mismo analizar el sentido y aplicación de algunas consideraciones éticas en torno al análisis de actividades como herramienta de investigación cualitativa además de analizar algunos aspectos metodológicos que conlleva este tipo de investigación para la movilización de los recursos personales.

Finalmente se concluye que el método del análisis de actividades permite y faculta al investigador la definición de un problema de investigación a lo largo del propio proceso de indagación, ponderando sucesivamente la eventual influencia del método en la definición de las hipótesis y de las fases de investigación sobre la dinámica de los objetivos de investigación.

\section{REFERENCIAS}

Argyris C. y Schon D. (1983). The Reflective Practitioner, How Professionals Think In Action.

Barbier J. M. (2000) (a). L'analyse de la singularité de l'action. CRF/ CNAM,
Paris, Puf. 192 - 265 p.- Coll. Éducation et Formation

Barbier J. M. (2004). (a) In Barbier J-M., Galatanu O. coord. Les savoirs d'action: une mise en mots des compétences - Paris L'Harmattan Collection Action et Savoir 1 partie chap 3 pp. 79 á 106.

Barbier J.M. (1996). L'analyse des pratiques: questions conceptuelles. In: Lánalyse des pratiques professionnelles - Ouvrage collectif coordonné par O. Fablet Et Cl. Blanchard-Laville.

Barbier J.M. (1999) (a). Practicas de formacion: evaluacion y analisis. Buenos Aires : Ed. Novedades educativas, Facultad de filosofia y letras de la Universidad de Buenos Aires.- 112 p.

Barbier J.M. (1999) (b). Tutoria y funcion tutorial: Algunas vias de analisis. in : Grupos y dispositivos de formacion.Ouvrage collectif .- Buenos Aires : Ediciones novedades educativas, Facultad de filosofia de Buenos Aires, 1999.- pp. 129-143.- (Coll. Formacion de formadores/Los documentos, $\mathrm{n}^{\circ} 10$.

Barbier J.M. Galatanu O. (2000). L'analyse de la singularité des actions: quelques outils conceptuels. In : CRFCNAM.- L'analyse de la singularité de l'action.- Paris, Puf.- pp. 13-51. Coll. Education et formation.

Barbier J-M , Galatanu O. (2004) (b). Savoirs, capacités, compétences, organisation des champs conceptuels In Barbier J-M., Galatanu O. coord. Les savoirs d'action : une mise en mots des compétences- Paris L'Harmattan Collection Action et Savoir rère partie chap 2 pp.31 à 78 .

Barbier J-M. Galatanu O. (2004) (a). Coordonnateurs Groupe Savoir d'Action du Cnam Les savoirs d'action : une mise en mots des compétences ? L'Harmattan- coll. Action et Savoir aout 2004 325p.

Barros Oscar, (1980). Investigación Operativa Análisis De Sistemas, Editorial Universitaria.

Beckhard Richard, Harris Reuben T. (1988). Transiciones organizacionales. Administración del cambio ( $2^{\mathrm{a}}$. ed.). USA; Adison - Wesley Iberoamericana. Belisle C. y Linard M. (1996). Quelles nouvelles compétences des acteurs de la formation dans le contexte des TIC. Education Permanente (127). 
Benson B. y Hughes J.A. (1983). The perspective of Ethnomethodology. London: Longman.

Blaug M. (1976). Kuhn versus Lakatos o paradigmas versus programas de investigación, en la historia de la Economía Pura. En: Revista Española de Economía. Madrid, 1976. p. 11.

Bruner Jerome (1991). Actos de significado, Alianza Editorial: Madrid.

Bruner Jerome, (1986). Realidad Mental y mundos posibles. Los actos de la imaginación que dan sentido a la experiencia, ed. Gedisa: Barcelona; trad. Beatriz López. 182 pp

Cortina Adela, (1994). Ética de la empresa: claves para una nueva cultura empresarial. Madrid: Trotta, 152 Pág. Cortina Adela, (2007). El concepto moderno de empresa ha de incluir necesariamente cuestiones éticas. [en línea] [08/10/o7] Disponible en: <http:// www.manosunidas.org/opinion/adela_cortina.htm>

Covarrubias Villa Francisco, (1995) (a). Las herramientas de la razón. La teorización potenciadora intencional de procesos sociales, ed. UPN: México; Col. Textos, No. 3, 277 pp.

Covarrubias Villa Francisco, (1999). La generación histórica del sujeto individual, Producción social de satisfactores y producción social de sujetos, Ed. UPN-Unidad Zamora-Colegio de Investigadores en Educación de Oaxaca: México; 215 pp.

Covarrubias Villa Francisco. (1995) (b). La teorización de procesos histórico-sociales. Volición, ontología y cognición científica, ed. UPN-SEP: México; Col. Textos, No. 4. 362 pp.

Covarrubias Villa, Francisco. (1998). Manual de Técnicas y Procedimientos de Investigación Social desde la Epistemología Dialéctica Crítica, Ed. UPN-Unidad 201 Oaxaca-Colegio de Investigadores en Educación: México; 146 pp.

Dagum C. (1978). Ideología y metodología de la investigación en la ciencia económica. En: Metodología y crítica económica: México. F.C.E., 1978. p. 93.

De Soto Domingo, (1975). Relección sobre el libro cuarto de las Sentencias acerca del dominio. Citado por Restituto Sierra Bravo, El pensamiento social y económico de la escolástica. Consejo Superior de Investigaciones Científicas.
Madrid, vol. 11, p. 627.

De Soto Domingo, (1975). Relección sobre el libro cuarto de las Sentencias acerca del dominio. Citado por Restituto Sierra Bravo, El pensamiento social y económico de la escolástica. Consejo Superior de Investigaciones Científicas. Madrid, vol. 11, p. 627.

Dejours Christophe, (1998). El Factor Humano. Asociación Trabajo y Sociedad. PIETTE. CONICET. (Buenos Aires: Lumen Humanitas).

Denison Daniel R. (1991). Cultura corporativa y productividad organizacional. Colombia; Fondo editorial Legis S.A.

Easlea Brian, (1977). La liberación social y los objetivos de la ciencia. México : Siglo XXI, 1977. p. 35. https:// www.iberlibro.com/buscar-libro/titulo/la-liberacion-social-y-los-objetivos-de-la-ciencia/autor/brian-easlea/

Echebarría K. (2000). La Administración Pública en la era del Management, Pensar lo Público, Longo y Zafra Eds., Unión Iberoamericana de Municipalistas - Esade, Granada, 52:53.

Edvinnson Leif y Malone Michael S. (2000). El Capital Intelectual, Como Identificar Y Calcular El Valor De Los Recursos Intangibles De La Empresa, Ediciones Gestión 2000 S.A, Barcelona, España.

French W.L. (1969). Organizacional Development: Objetives, Assumptions and Strategies, por los regentes de la University of California. Reimpresión de la California Management Review, vol XII, núm. 2, pág. 26.

French y Bell, (1990). Organizational Development: Behavioral science interventions for organization improvement; Englewood Cliffs, N.J. Prentice-Hall).

Frohman, Sashkin y Kavanagh M.J. (1976). Action-research as applied to organization development. Organization and Administrative Sciences, vol. 7, no. 1 and 2 .

Garza Rosa María y Susana Levanthal. (2000) (3). Aprender cómo aprender, ed. Trillas: México; $160 \mathrm{pp}$.

Glasersfeld E. Von (1993, ed. alemana de 1981). Introducción al constructivismo radical, en Watzlawick, P. et al. La realidad inventada. Barcelona: Gedisa.

Habermas Jürgen, (1996). La Lógica De Las Ciencias Sociales, Editorial Tecnos 
S.A, Tercera Edición. Primera Edición en 1988.

Hammersley M. y Atkinson P. (1983). Ethnography; Principals in practice (reprinted 1992) Routledge: London.

Hampson P.J. y Morris P.E. (1996). Understanding cognition. Cambridge, MA: Blackwell Publishers Inc.

Hanna David P. (1990). Diseño de organizaciones para la excelencia en el desempeño. Addison-Wesley Iberoamericana. México; Sisa técnicos de edición S.A.

Hernández S., Roberto Fernández C. Carlos., Baptista L Pilar, (2006). Metodología de la Investigación. $3^{\underline{a}}$ edición. Bogotá: Mc Graw Hill, 420 p.

Johnson-Laird P. (1983). Mental models. Cambridge: Cambridge University Press.

Johnson-Laird, P. (1990). Mental models, en Posner, M. (ed.), Foundations of cognitive science. Cambridge, MA: MIT Press, pp. 469-499.

Kemmis E. y McTaggart, (1998). Cómo Planificar la Investigación Acción. Barcelona: Laertes. 199p.

Kliksberg Bernardo, (1989). Gerencia Pública en tiempos de incertidumbre, INAP, Madrid.

Kuhn Thomas S. (2004). La estructura de las revoluciones científicas. Fondo de Cultura Económica, México. p. 318. https://materiainvestigacion.files.wordpress.com/2016/05/kuhn1971.pdf

Kuhn, Thomas S. (2004). La estructura de las revoluciones científicas. México : Fondo de Cultura Económica, p. 269.

Landier Hubert, (1992). Hacia la empresa inteligente. España; Ediciones Deusto S.A.

Le Boterf G. (2001). Ingeniería de las competencias. Barcelona. Gestión 2000 Le Boterf G. (1998). L'ingénierie des compétences. Editions d'Organisation. Le Boterf G. (2001). Construire les competences individuelles et collectives." Paris: Editions d'organisation.

Levy-Leboyer, C. (1995). Gestión de competencias. Gestión 2000.

Limone Aquiles y Cademártori David, (1998).a Empresa: Una Red De Transformaciones, Editorial Jurídica Conosur
Lincoln Y. y Denzin N. (1994). The Fifth Moment. Handbook of Qualitative Research. Eds. Denzin and Lincoln. Thousand Oaks: Sage Publications. 575-586.

Martínez Delgado, A. (1995). Constructivismo, ¿una vuelta a los principios filosóficos del positivismo? Comunicación, Lenguaje y Educación, 28, pp. 5-13.

Martínez Delgado, A. (1998). Ideas previas: experimentación acerca de ideas arraigadas e ideas inducidas sobre fracciones. Suma, 28, pp. 59-70.

Piaget J. (1975/1983). La psicogénesis del conocimiento y su significado epistemológico, en Chomsky, N. y Piaget J. Teorías del lenguaje. Teorías del aprendizaje. Barcelona: Crítica.

Pieró José M. (1986). Psicología de la organización. Universidad nacional de educación a distancia.

Popper Karl R. (1994). En Busca De Un Mundo Mejor, Editorial PAIDOS.

Pourtois Sean-Pierre, Desmet Huguette, (1992). Epistemologia E Instrumentacion En Ciencias Humanas, Editorial Herder.

Sanguino R. (2003). La Gestión Del Conocimiento. Su Importancia Como Recurso Estratégico Para La Organización",[en línea] 5campus.org, Marketing http:// www.5campus.org/leccion $/ \mathrm{km}$.

Schön D. A. (1983). The Reflective Practitioner; New York, Basic Books. Inc.

Senge Peter (1995). La Quinta disciplina: las organizaciones que aprenden. Editorial Mc Graw - Hill.

Siegel, S. Castellán, N., 1985. Estadística no Paramétrica, México. $4^{\circ}$ Edición. McGraw-Hill Interamericana Editores SA.; Cita a Bradley, 1968.

Spencer L. (1993). Competence at Work, Models for Superior Performance. Editorial John Wiley \& Sons, Inc.

Wertsch James V. (1988). Vygotski y la formación social de la mente, ed. Piadós: Barcelona; Col, Biblioteca cognición y desarrollo humano/17. trad. Javier Zenón y Montserrat Cortés. 264 pp. 